\title{
Texture Dependent Strain Rate Sensitivity of Ultrafine-grained Aluminum Films
}

\author{
Ehsan Izadi and Jagannathan Rajagopalan ${ }^{*}$ \\ Department of Mechanical and Aerospace Engineering \\ School for Engineering of Matter, Transport and Energy, \\ Arizona State University, Tempe 85287, USA.
}

\begin{abstract}
:
We investigated the strain rate sensitivity (SRS) of freestanding ultrafine-grained aluminum films with similar thickness $(\sim 240 \mathrm{~nm})$ and mean grain size $(\sim 275 \mathrm{~nm})$ but highly dissimilar texture. For (110) textured bicrystalline films, flow stress increased by $14 \%(m=0.017)$ as the strain rate was varied from $7 \times 10^{-6} / \mathrm{s}$ to $5 \times 10^{-3} / \mathrm{s}$. In contrast, for non-textured films the flow stress increased by more than $90 \%(m=0.103)$ over a similarstrain rate range. The drastic difference in SRS can be explained by texture-induced changes in deformation mechanisms of the films, as revealed by in situ TEM straining experiments.
\end{abstract}

Keywords:Strain rate sensitivity; texture; ultra-fine grained microstructure; thin films; TEM

${ }^{*}$ Corresponding author. Email: jrajago1@asu.edu 
Ultrafine-grained (UFG) and nanocrystalline (NC) metalsexhibit a number of appealing mechanical properties such as high strength and increased wear resistance[1].Metallic thin films withUFG/NCmicrostructuresare employed innumerous applications includingstructural coatings, interconnects in semiconductor devices, in thermomechanical applications and as structural and electrode components in micro-electro-mechanical systems (MEMS) [2-4]. In many of these applications, and particularly in MEMS, the metallic films may be subjected to a wide range of loading rates. For instance, in MEMS based high frequency oscillators or thermomechanical switches the actuation times can vary from microseconds to milliseconds.

It is known that the mechanicalresponseofUFG/NC metal films depends on the rate at which they are deformed.Therefore, in order to gain reliable operation of MEMS, the mechanical behavior of metal films over various loading rates needs to be understood[4,5].Strain rate sensitivity (SRS) studies of thin metal films have typicallyfocused on mechanical behavior at ratesbetween $10^{-6} / \mathrm{s}$ and $10^{-3} / \mathrm{s}[6-9]$, butmore recentlya wider range of strain rates[4,10-12] have been explored. SRS studies of UFG/NC metalshave been mainly directed towards elucidatingthe effect of mean grain size on their rate dependent mechanical response. These studies have shown thatacross a wide range of metalsSRS increases and activation volume decreases $[6,7,10,13]$ as the grain size becomes finer.

But in addition to the mean grain size, texture is also known to significantly influence the deformationbehavior of UFG/NC metal films. Torre et al. have shown that differences in texture lead to variation in the yield stress and ultimate tensile stress ofNC Ni foils[14].Similarly, experiments on nanoscale Al films with similar mean grain size and thickness but different textures have revealed significant differences in flow stress and Bauschinger effect $[15,16]$. However, the effect of texture on the SRSof metallic films has not been systematically investigated so far.

In this study, we investigated the SRS of two sets of nanoscale Al films with nearly identical thickness ( $240 \mathrm{~nm})$ and mean grain size (275-285 nm), but substantially different textures.Our 
results unambiguously show thatSRS of ultrafine-grained Al filmsis strongly dependent on film texture. Films with a strong (110) out-of-plane texture and composed only of two in-plane grain variants (bicrystalline films) show significantly smallerSRS compared to films with a random orientation of grains (non-textured films). The flow stress of the bicrystalline films increasedby $14 \%$ when the strain rate was increased from $7 \times 10^{-6} / \mathrm{s}$ to $5 \times 10^{-3} / \mathrm{s}$. In contrast, the flow stress of non-textured films increased byover90\% over a similar strain rate range.

The bicrystalline Al film (labeled as textured film) and the non-textured Al film were synthesized by carefully controlling the deposition conditions. To obtain the textured film, the native silicon dioxide layer on a Si (001) wafer was removed through hydrofluoric acid etching and the wafer was immediately transferred to the sputtering chamber to avoid regrowth of the oxide layer. Al was then deposited on the bare silicon wafer using DC magnetron sputtering, which resulted in heteroepitaxial growth with the following relationship: Al(110)//Si(001), Al[001]//Si[110] and $\mathrm{Al}(110) / / \mathrm{Si}(001), \mathrm{Al}[001] / / \mathrm{Si}[110][17]$. Thus, this film consists of just two grain families with (110) out-of-plane texture which are rotated $90^{\circ}$ in plane with respect to each other. The nontextured film was obtained using a similar process except that itwas sputter deposited on Si (001) wafers with the native silicon dioxide layer intact. The oxide layer disrupts the epitaxial growth of Al, leading to a film with random orientation of grains. Both the textured film and non-textured film wasdeposited to a thickness of $240 \mathrm{nmat} 5.5 \mathrm{~nm} / \mathrm{min}$. Thechamber base pressure during deposition of the textured and non-textured film was $8 \times 10^{-8}$ Torr and $3 \times 10^{-}$ ${ }^{7}$ Torr, respectively.

The microstructure of the films was examined through transmission electron microscopy (TEM) and x-ray diffraction (XRD). Based on plan-view TEM images the textured Al film had a mean grain size of $275 \mathrm{~nm}$ and the non-textured film had a mean grain size of $285 \mathrm{~nm}$ (Fig. 1). The images also indicated a columnar grain structure with one grain traversing the thickness of the film.Dog-bone shaped freestanding samples were then co-fabricated with micro-electromechanical systems (MEMS) based tensile testing devices (Fig. 2a) using microfabrication techniques outlined in [18].The MEMS devices have built-in gauges to track the sample 
deformation. Apiezoelectric actuator (Physik Instrumente)was used to load the devices and a CMOS camera (Thor Labs) was used to acquire images of the gauges during the experiments. A custom MATLAB ${ }^{\mathrm{TM}}$ program, which tracks prescribed features across a series of images using cross-correlation techniques, was used to measure the displacement of the gauges and thus the sample strain.

A miniature s-beam load cell (Futek), arranged in series with the MEMS device, measured the total force $\left(F_{\text {tot }}\right)$ on the device (Fig. 2 b). As evident from the equivalent mechanical model of the device, $F_{\text {tot }}=F_{S}+F_{U}+F_{A}$, where $F_{S}$ is the force on the sampleand $F_{U}$ and $F_{A}$ are the force on the $U$-beams and the Alignment beams, respectively. The combined stiffness $(K)$ of the U-beams and Alignment beams was measured in a separate experiment after the sample had fractured. $K$ multiplied by the displacement $(x)$ gives $F_{U}+F_{A}$, from which $F_{S}$ and thus the stress on the sample was obtained.

In all the experiments, loading was along the [001] direction of the Al[001]//Si[110] grain family for the textured film and along an arbitrary direction for the non-textured film. To calculate the uncertainty in stress measurements, a range of loads were applied on the load cell and the fluctuations in force were recorded over time for each load. Based on the load cellfluctuations, the uncertainty in the calculated stress was less than $10 \mathrm{MPa}$ for all the samples.Similarly, for a prescribed displacement, images of the gauges were recorded over a period of time and analyzed. Based on the analysis, the error in strain measurement was found to be less than $0.003 \%$. There was also an 10 $\mathrm{nm}$ variation in the thickness of both the textured and nontextured film across the wafer. Both the load cell fluctuations and the variation in the film thickness were taken into account for calculating the error in the flow stress of the films.

The Al films were under compressive stress in the as-deposited state and hence the freestanding samples buckled when they were released from the Si substrate.Because the stress for buckling along the length is very low $(0.1 \mathrm{MPa})[16]$, the samples are almost macroscopically stress-free before loading.In addition, to eliminate possible variation in 
mechanical behavior that can arise from sample size effects, we ensured that all the samples from both the textured and non-textured film had identical dimensions (effective gauge length $375 \mu \mathrm{m}$, width $75 \mu \mathrm{m}$ ). Thus, by ensuring uniformity in the fabrication process, mean grain size, and sample dimensions, we were able to isolate the effect of texture on the strain-rate dependent behavior of the Al films.

Figure 3 shows the stress-strain response of the textured and non-textured filmat different strain rates. The corresponding durations of the experiments varied from less than 2 seconds at the fastest rate to more than 2000 seconds at the slowest rate. As evident from Fig. 3a, the stress-strain response of the textured film did not vary significantly with strain rate. At all rates, aninitial linear response was followed by a gradual elastic-plastic transition (microplastic regime) that is typical of UFG and NC metals.To quantify the strain rate effect, we calculated the stress at $0.9 \%$ strain $\left(\sigma_{0.9 \%}\right)$ at different rates and used it as a measure of flow stress. $\sigma_{0.9 \%}$ was chosen as a measure of flow stress because macroscopic plasticity had set in the samples before $0.9 \%$ strain and there was little subsequent strain hardening. Another consideration was thatthe samples failed around $1 \%$ strain at the highest strain rate. For thetextured filmflow stressincreased from $302 \mathrm{MPa}$ to $342 \mathrm{MPa}(14 \%$ increase) as the strain rate increased from $6.9 \times 10^{-6} / \mathrm{s}$ to $5.3 \times 10^{-3} / \mathrm{s}$.

In contrast, the non-textured film (Fig. 3b) showed considerable difference in stress-strain response over a similar range of strain rates. Flow stress $\left(\sigma_{0.9 \%}\right)$ increased from $243 \mathrm{MPa}$ to 473 $\mathrm{MPa}$ as the strain rate was increased from $6.8 \times 10^{-6} / \mathrm{s}$ to $6.7 \times 10^{-3} / \mathrm{s}$.In addition, during theelastic-plastic transition the stress-strain slope wassignificantly higher at the higher strain rates. However, once macroscopic plasticity set inlittle hardening was observed, irrespective of the strain rate. Thus, while the flow stress was sensitive to the strain rate, the rate of strain hardening was not.To quantitatively compare the stress-strainresponse of thetextured and nontextured film, we calculated the strain rate sensitivity(SRS) exponent $(m=d \log (\sigma) / d \log (\dot{\varepsilon}))$ for both the films (Fig.3c). mfor the non-textured film was 0.103 , more than sixtimes higher compared to the textured film $(m=0.017)$. 
We note that the Young's modulusobtained for both the films is less than that of bulk Al $(E=69$ GPa). The primary reason for this lower $E$ is that ourfreestanding sampleshave a notable curvature (buckling) along the widthas well.Thus, the deformation is not uniform during the initial stages of loading and the actual sample cross-section being deformed is smaller than the nominal cross-section assumed for stress calculation. Hence, the stress during the initial phase of loading is underestimated, which results in a lower E.Tensile load-unload experiments on similar Al films indeed show that the modulus obtained from unloadingis higher and significantly closer to the bulk value (Supplementary Figure 1).

Previous studies show that NC and UFGface-centered cubic (FCC) metals exhibit strain rate sensitivities that are anorder of magnitude larger than coarse-grained FCC metals, which usually have mof 0.001 to $0.005[19-21]$. UFG Al, for example, has been shown to exhibitm values ranging from 0.02 to $0.2[22-26]$ at strain rates comparable to those used in this study. Similarly in NC Al films that undergo discontinuous grain growth, mvalues ranging from 0.035 to 0.14 have been reported[5].

Typically, $m$ values progressively increase as the grain sizeis reduced from the microcrystalline to the NC regime[6,27-29]. This grain size dependence has been attributed to the changes in deformation mechanisms that accommodate plasticity[29].In coarse-grained metals, the ratecontrolling process is the cutting of forest dislocations, which results in a low SRS. In contrast, for NC and UFG metals a primary rate-limiting process is the interaction between dislocations and grain boundaries, which serve as obstacles to dislocation motion[30]. Hence, as the density of grain boundaries increases with decreasing grain size, the rate-dependence of deformation behavior becomes more pronounced.

Our resultsshow that UFG metal films withdifferent textures can have considerable differences in SRS, even when their mean grain size and thickness are nearly identical. This suggests that texture plays an equally important role as grain size in determining the rate sensitivity and deformation mechanisms of UFG metal films. 
To obtain insight into the deformation mechanisms that are active in the textured and nontextured films, we performed quasi-static in situ straining experiments on these films in a JEOL 2010F TEM. The experiments were performed under a low intensity, $200 \mathrm{kV}$ electron beam to minimize beam-induced artifacts during in situTEM deformation [31]. When the textured film began to deform plasticallythere was notable dislocation activity, anda few grains showed changes in contrast (indicative of out-of-plane grain rotation) when the strain was increased. However, there were no time-dependent changes in grain contrastor motion of bend contours when the strain was held constant (Supplementary Figure 2). It is worth noting that previousin situTEM studies of such textured Al films have shown similar results[15,31].

The behavior of the non-textured film, in contrast, was qualitatively different, witha larger fraction of grains showinginstantaneous changes in contrast when strain was increased (Fig. 4). More importantly, the grainsalso underwent gradual changes in contrastover time, often accompanied by motion of bend contours,even when the strain was held constant.Since the grain orientations were continuously changing and most grains were away from the Bragg condition it was difficult to observe dislocation activities in the non-textured film. Nevertheless, the time-dependent grain orientation changes suggest that diffusive relaxation processes could be active in addition to dislocation plasticity.

The strain rate experiments in conjunction with the qualitative in situ TEM experiments point to the following picture of deformation in the textured and non-textured film. In the textured (bicrystalline) film, the initial deformation is relatively homogeneous because elastic modulusfor the two sets of grains along their loading directions $(<100\rangle$ and $\langle 110\rangle)$ is quite similar (63.7 GPa and $72.5 \mathrm{GPa}$, respectively). Thus, the elastic strain mismatch between the grains is smalland since they have the same out-of-plane orientation little or no grain rotation is required to maintain strain compatibility.

After the initial elastic regimethe film undergoes microplastic deformationas relatively larger grains yield first and are followed by progressively smaller grains. However, both sets of 
grainshavesufficient slip systems to accommodate the imposed deformation and all the active slip systemsin both sets of grains have the same Schmid factor $(s=0.408)$. In effect, plastic anisotropy is nearly eliminated and hence the plastic strain mismatch between the grains is quite small.This againreduces the need forgrain rotation to accommodate the deformation.

In contrast, deformation of the non-textured film is considerablymore heterogeneous. Because the grains are randomly oriented, there ismore variation in elastic modulus (63.7-76 GPa) and hence higher elastic strain mismatch between the grains. More importantly, themaximum Schmid factor (s)of the grains varies from 0.27 to 0.5 . Therefore, plastic anisotropy $\left(s_{\max } / s_{\min }\right)$ in the non-textured film is significantly more pronounced compared to the textured film. As a result, the plastic strain mismatchbetween adjoining grains is substantially higher,and thegrains need to bend or rotate with respect to each other to maintain strain compatibility.

If the applied strain rate is low, the grains can relax the stress arising from elastic/plastic incompatibility through diffusive processes.But at higher strain rates suchrelaxation is not possible and thus the macroscopic stress-strain response becomes highly rate sensitive. Note that even though the experiments are performed at room temperature, the homologous temperature ( $\left.T / T_{\text {melting }}\right)$ for Al exceeds 0.3. And since the thin film samples have a large surface to volume ratio, surface diffusion, which has lower activation energy compared to bulk diffusion, can be significant and lead to stress relaxation.

In this context, it is also worth noting that at lower strain rates the non-textured film exhibits lower flow stress than the textured film, whereas the opposite is true at higher strain rates. One possible explanation for this trend is that at lower rates the non-textured film accommodates thedeformation through both dislocation plasticity and diffusive processes, which leads to lower stresses compared to the textured film. At higher rates, both the films are likely to accommodate the deformation primarily through dislocation plasticity. And sincethe grains in the textured film have a relatively high average Schmid factor $(s=0.408)$, the flow stress of the textured film is lower comparedto the non-textured film. 
Overall, the primary finding from our experiments is that the deformation behavior of UFGaluminum films is highly dependent on their texture. The texture-induced changes in deformation mechanisms can introduce substantial changes in strain rate sensitivity, even in films that have nearly identical mean grain sizes and thickness. Thus, it is important to consider both the effect of texture as well as grain size in inferring the deformation mechanisms of UFG metals from strain rate sensitivity measurements.

\section{Acknowledgements}

This project was funded by the National Science Foundation (NSF) grants CMMI 1400505 and DMR 1454109. The authors would like to gratefully acknowledge the use of facilities at the John M. Cowley Centre for High Resolution Electron Microscopy and the Centre for Solid State Electronics Research at Arizona State University.

\section{References}

[1] M.A. Meyers, A. Mishra, D.J. Benson, Prog. Mater. Sci. 51 (2006) 427-556.

[2] R.G. Polcawich, J.S. Pulskamp, D. Judy, P. Ranade, S. Trolier-McKinstry, M. Dubey, IEEE Trans. Microw. Theory Tech. 55 (2007) 2642-2654.

[3] N. Ledermann, P. Muralt, J. Baborowski, S. Gentil, K. Mukati, M. Cantoni, A. Seifert, N. Setter, Sens. Actuators Phys. 105 (2003) 162-170.

[4] I. Chasiotis, C. Bateson, K. Timpano, A.S. McCarty, N.S. Barker, J.R. Stanec, Thin Solid Films 515 (2007) 3183-3189.

[5] D.S. Gianola, D.H. Warner, J.F. Molinari, K.J. Hemker, Scr. Mater. 55 (2006) 649-652.

[6] R. Schwaiger, B. Moser, M. Dao, N. Chollacoop, S. Suresh, Acta Mater. 51 (2003) 51595172.

[7] Y.M. Wang, A.V. Hamza, E. Ma, Appl. Phys. Lett. 86 (2005) 241917.

[8] R.D. Emery, G.L. Povirk, Acta Mater. 51 (2003) 2067-2078.

[9] R.D. Emery, G.L. Povirk, Acta Mater. 51 (2003) 2079-2087.

[10] Z. Jiang, X. Liu, G. Li, Q. Jiang, J. Lian, Appl. Phys. Lett. 88 (2006) 143115.

[11] K. Jonnalagadda, N. Karanjgaokar, I. Chasiotis, J. Chee, D. Peroulis, Acta Mater. 58 (2010) 4674-4684.

[12] N.J. Karanjgaokar, C.-S. Oh, J. Lambros, I. Chasiotis, Acta Mater. 60 (2012) 5352-5361.

[13] H.S. Kim, Y. Estrin, Acta Mater. 53 (2005) 765-772.

[14] F. Dalla Torre, H. Van Swygenhoven, M. Victoria, Acta Mater. 50 (2002) 3957-3970.

[15] J. Rajagopalan, C. Rentenberger, H. Peter Karnthaler, G. Dehm, M.T.A. Saif, Acta Mater. 58 (2010) 4772-4782.

[16] J. Rajagopalan, M.T.A. Saif, J. Mater. Res. 26 (2011) 2826-2832. 
[17] H. Niwa, M. Kato, Appl. Phys. Lett. 59 (1991) 543-545.

[18] J.H. Han, J. Rajagopalan, M.T.A. Saif, in:, 2007, p. 64640C-64640C-8.

[19] Y.M. Wang, E. Ma, Mater. Sci. Eng. A 375-377 (2004) 46-52.

[20] G. Mohanty, J.M. Wheeler, R. Raghavan, J. Wehrs, M. Hasegawa, S. Mischler, L. Philippe, J. Michler, Philos. Mag. 0 (2014) 1-18.

[21] V. Maier, K. Durst, J. Mueller, B. Backes, H.W. Höppel, M. Göken, J. Mater. Res. 26 (2011) 1421-1430.

[22] M.S. Mohebbi, A. Akbarzadeh, B.H. Kim, S.-K. Kim, Metall. Mater. Trans. A 45 (2014) 54425450.

[23] J. May, H.W. Höppel, M. Göken, Scr. Mater. 53 (2005) 189-194.

[24] A. Böhner, V. Maier, K. Durst, H.W. Höppel, M. Göken, Adv. Eng. Mater. 13 (2011) 251255.

[25] D.T. Read, Y.-W. Cheng, R.R. Keller, J.D. McColskey, Scr. Mater. 45 (2001) 583-589.

[26] J.M. Wheeler, V. Maier, K. Durst, M. Göken, J. Michler, Mater. Sci. Eng. A 585 (2013) 108113.

[27] F. Dalla Torre, P. Spätig, R. Schäublin, M. Victoria, Acta Mater. 53 (2005) 2337-2349.

[28] Y. Wang, A. Hamza, E. Ma, Acta Mater. 54 (2006) 2715-2726.

[29] M. Dao, L. Lu, R.J. Asaro, J.T.M. De Hosson, E. Ma, Acta Mater. 55 (2007) 4041-4065.

[30] H. Van Swygenhoven, P.M. Derlet, A.G. Frøseth, Acta Mater. 54 (2006) 1975-1983.

[31] R. Sarkar, C. Rentenberger, J. Rajagopalan, Sci. Rep. 5 (2015) 16345. 


\section{Figure captions}

Figure 1: (a) Bright-field TEM image of the textured Al film with a mean grain size of $275 \mathrm{~nm}$. Selected area diffraction (SAD) of the film showing an (110) out-of-plane texture with two inplane variants rotated $90^{\circ}$ with respect to each other (inset).(b) Bright-field TEM image of the non-textured Al film with a mean grain size of $285 \mathrm{~nm}$. Selected area diffraction (SAD) of the film showing the lack of texture (inset).

Figure 2: a) MEMS device for performing constant strain rate experiments on freestanding metal film samples. The nominal strain on the sample is obtained by tracking the displacement of gauges G1 and G2. b) Equivalent mechanical model of the MEMS device. The U-beams and Alignment beams are in parallel with the sample and hence their displacement $(x)$ is the same. The load cell is arranged in series with the MEMS device.

Figure 3: (a) Stress-strain response of the textured film at different strain rates. (b) Stress-strain response of the non-textured film at different strain rates. (c) Log-log plot of flow stressversus strain rate for the textured and non-textured film. The error bars indicate the bounds of uncertainty in flow stress arising from both film thickness variation and fluctuations in the load cell measurements.

Figure 4: (a) Bright field TEM image of a non-textured film at $1.19 \%$ strain. (b) Image of the same area at $1.3 \%$ strain. The numbers in red identify a sampling of grains that showed a drastic change in contrast (indicative of orientation change). Arrows point to new bend contours that resulted from the strain increment. 


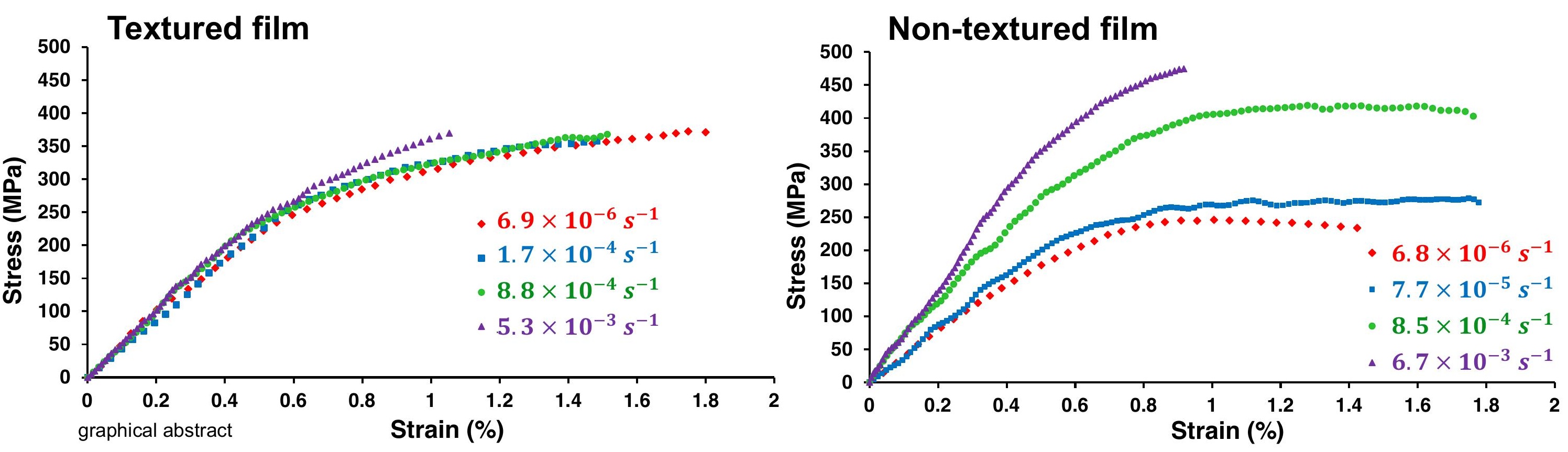




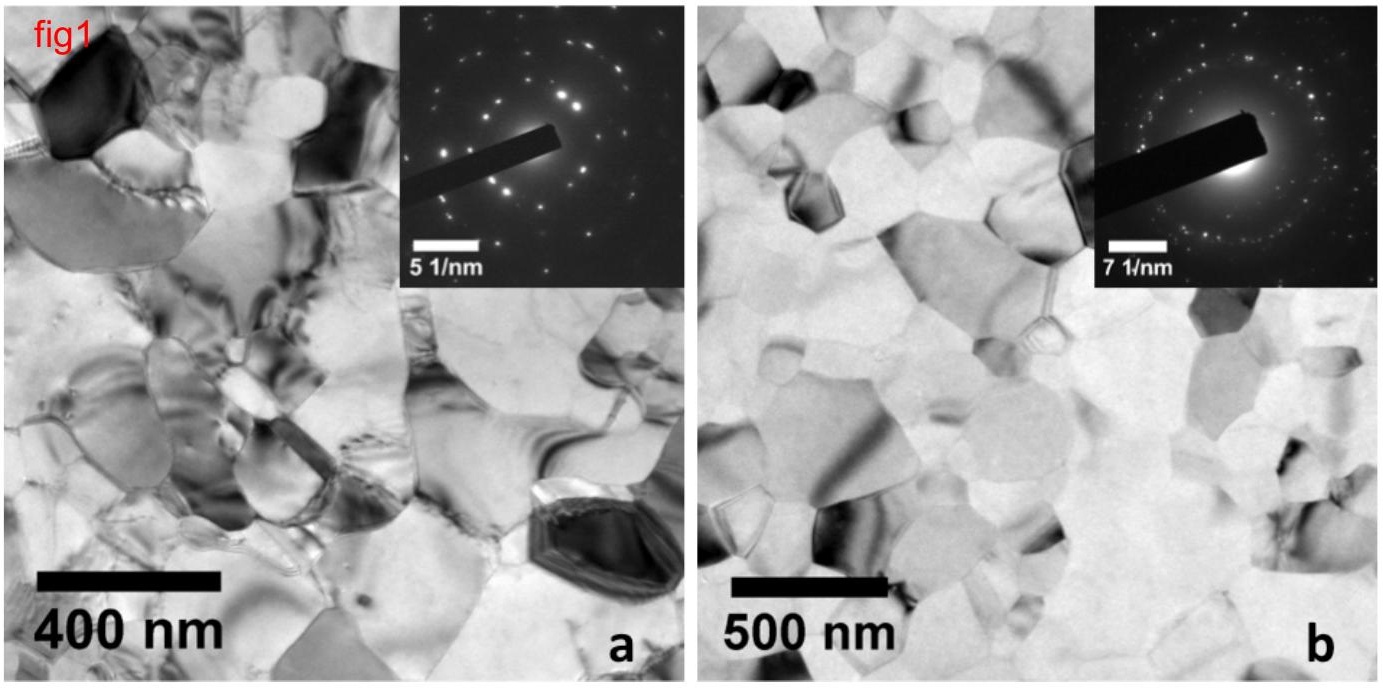




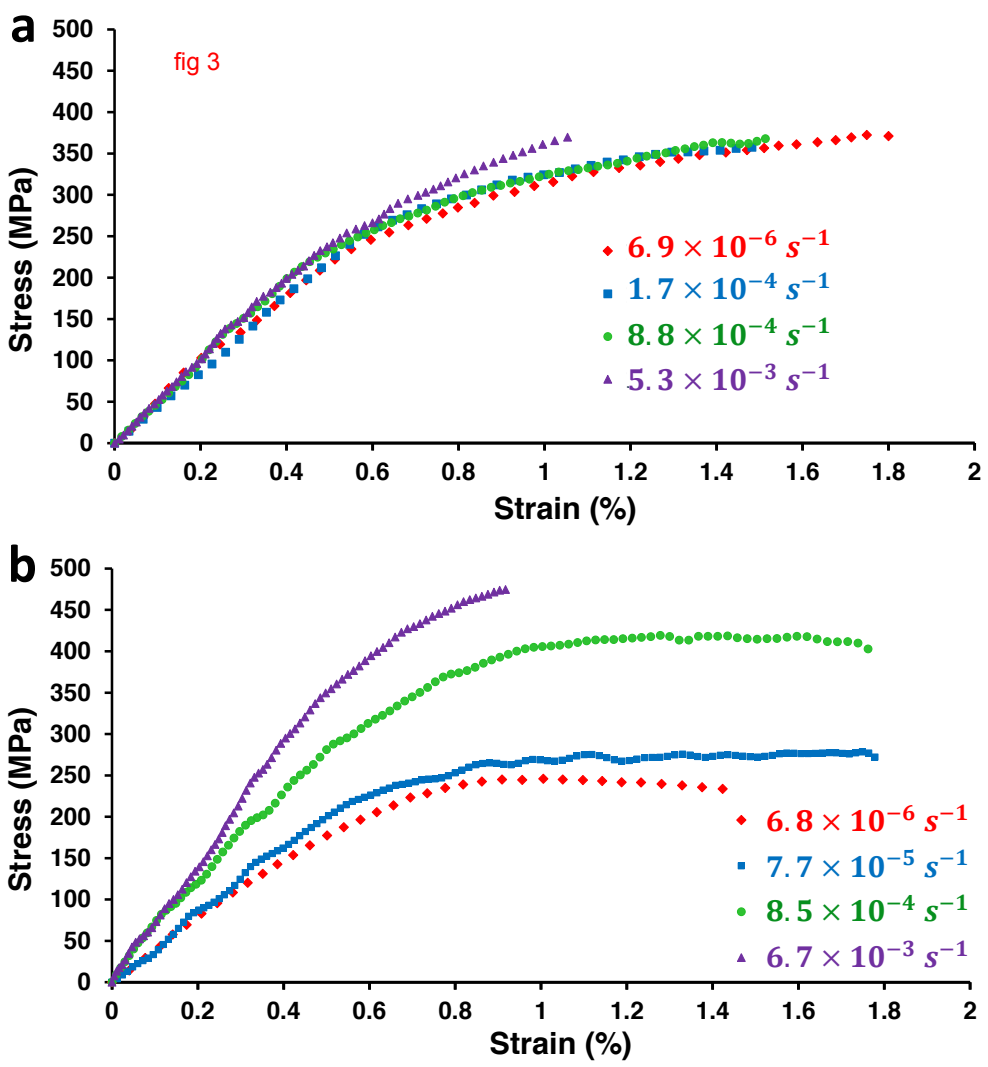

C

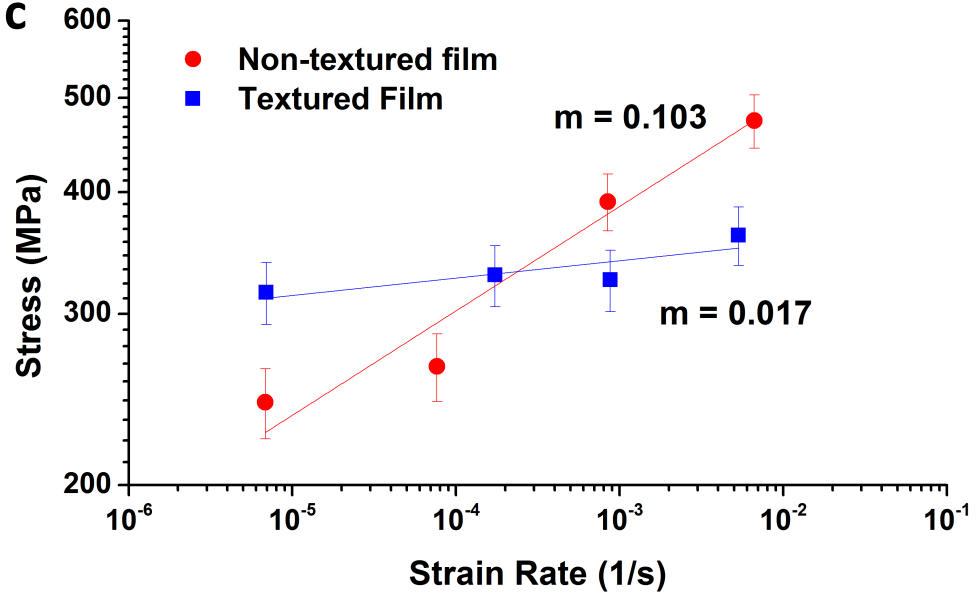




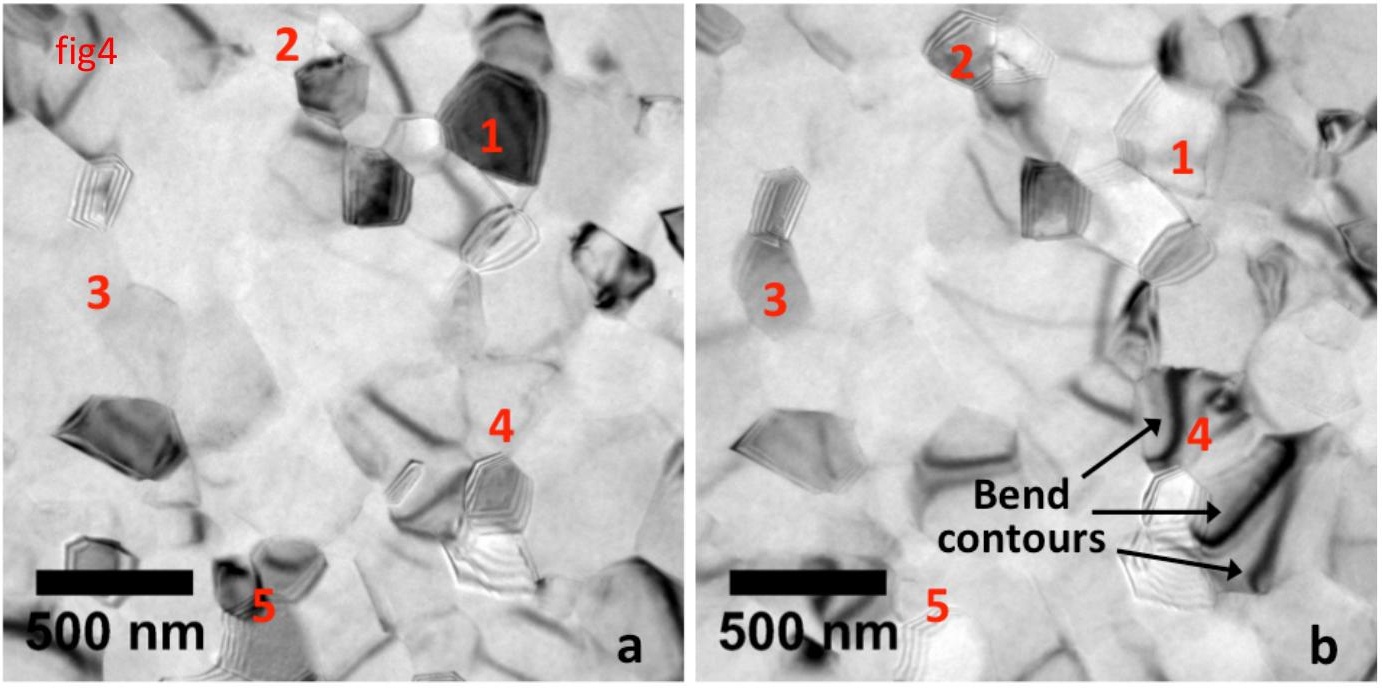

